\title{
Radiosurgery for arteriovenous malformations in eloquent region of the brain
}

\author{
Wen-Yuh Chung \\ Department of Neurosurgery, Neurological Institute, Taipei Veterans General Hospital, National Yang-Ming University, Taipei, \\ Taiwan
}

\author{
Address for correspondence: \\ Dr. Wen-Yuh Chung, \\ Department of Neurosurgery, \\ Neurological Institute, Taipei \\ Veterans General Hospital, No. 201, \\ Sec. 2, Shih-Pai Rd., 112, Taipei, \\ Taiwan. \\ E-mail:wychung@vghtpe.gov.tw
}

PMID: 19934549

DOI: $10.4103 / 0028-3886.57788$

For a neurosurgeon, one of the most difficult tasks is to manage arteriovenous malformations (AVMs) in eloquent locations. The difficulty is two folds, namely, when and how to manage the malformation. Many neurosurgeons agree with the fact that management of cerebral AVM is a matter of all-or-none issue; once you start, you cannot stop half way. The delicate AVM hemodynamics will be changed after management; moreover, even a subtle change may result in catastrophic hemorrhage and irreversible severe neurological deficits. In fact, some AVM might be more 'benign' than previously considered. The 'ARUBA' study is now underway in spite of controversies in many aspects. ${ }^{[1]}$ We do not know if any conclusion will be drawn after completion of the study, but we should bear in mind that observation (waitand-see) is another option of management for AVM in eloquent locations.

Presently available management options are microsurgery, radiosurgery and embolization. In some cases a combined strategy with embolization and radiosurgery aiming to reduce the risk of hemorrhage or to lessen neurological symptoms with acceptable risks is plausible. ${ }^{[2]}$ Nevertheless, each treatment modality is inherited with pro and con. As reviewed in the article by Javalkar et al., ${ }^{[3]}$ microsurgical excision of AVM in eloquent region reported by experts is associated with a significant morbidity (6.1 to $11 \%$ ) and, occasionally, mortality (0.6 to $5 \%)$. On the contrary, radiosurgery is associated with relative low morbidity ( 3 to $5.3 \%$ ) and seldom reported radiosurgery-related mortality. Embolization is associated with high morbidity unless the embolization is only aimed for partial treatment to decrease bleeding risk from fistular component or aneurysmal sac of AVM. Radiosurgery, being different from the other two management options, with gradually evolving radiation effects to obliterate AVM nidus gives brain tissues a wide time window to adopt new hemodynamics or tolerate side effects that may occur occasionally. Nevertheless, management decision tends to be biased towards modalities available 'in-house'. Neurosurgeons in individual center did choose the modality as they are good at.

This article by Javalkar et al., ${ }^{[3]}$ had similar results reported previously. The overall outcome seemed better than the results of microsurgery or embolization alone. However, it is still unclear regarding the issues, when and how to manage, and dose selection in radiosurgery. Based on our experience, we had several comments on these subjects. 


\section{Patient Selection and Angioarchitectures}

No intervention is needed for AVM without symptom, previous hemorrhage or 'benign' angioarchitectures. In general, the risk factors of hemorrhage, such as deep-seated or peri-ventricular location, outflow obstruction of draining veins, venous ectasia/stenosis/ hypertension and presence of intranidal aneurysm or pseudoaneurysm, prompt us to treatment. Other kinds of angioarchitecture, e.g. compactness of nidal vessel or sign of the hemodynamic decompensation of AVM, should also be taken into consideration. For AVM neighboring ventricles or cisterns, higher dose irradiation can be prescribed and larger volume AVM can be treated with the benefit of nearby CSF protection. AVM with a volume larger than $40 \mathrm{~mL}$ was suggested to treat by staged radiosurgery or combination with embolization.

\section{The Dose Suggested for Arteriovenous Malformations in Eloquent Area}

For eloquent location AVM, it is a challenge to make a balance among prescribed irradiation dose, cure rate and complication rate. The low morbidity in this series may indicate a good balance among the aforementioned concerns. ${ }^{[3]}$ In our experience, 16 Gy seems to be the minimal effective marginal dose to initiate the radiation effects on endothelium of AVM nidus for obliteration. ${ }^{[4]}$ The normal surrounding brain tissues seemed to tolerate radiation as high as $15 \mathrm{~Gy}$ in a single fraction irradiation. In addition, we tailored the dosimetry as conformal as possible by using multiple shots and achieving the mean dose to the targeted volume in a range of $22 \mathrm{~Gy}$ to $25 \mathrm{~Gy}$. When second radiosurgery is needed four years after first gamma knife radiosurgery, we think a same marginal dose could be prescribed with an acceptable risk of adverse reaction. With this as a background, AVM patients with nidal volume larger than $40 \mathrm{~mL}$ may gain the most by staged radiosurgery as far as cure rate and complication rate are concerned.

\section{Staged Radiosurgery}

Several reports have discussed the strategy in treating large AVMs, including spatially fractionated (volumestaged), temporary fractionated and hypofractionation radiosurgery. Our results convinced that volume-staged radiosurgery is a safe and practical treatment for patients with extra-large cerebral AVMs. ${ }^{[5]}$ If the nidus is compact without much intervening brain tissue, we can use two independent dose plans to complete treatment. In the case of AVM with less compact nidus, it would be better to perform prospective dose plan to cover the whole nidus, and to decrease adverse radiation effect to normal brain tissue. Our method overcomes the difficulty in avoiding unexpected injury from hot spots between two independent dose plans. However, the common drawback of staged radiosurgery is the long waiting time for nidus to be totally obliterated.

\section{Peri-Radiosurgery Embolization}

Trans-arterial embolization performed before or after radiosurgery has earned higher appraisal recently. The purpose of embolization is to decrease impending hemorrhage risk and accelerate the obliteration of remaining nidus. In case of a patient with large AVM in whom there is a big arteriovenous shunt after expected latency, the patient actually benefited from micro-catheter embolization to obliterate the associated shunt and reduced hemorrhage risk. ${ }^{[5,6]}$ We suggest that one should complete the staged radiosurgery first in cases involving large AVMs, and then obtain a follow-up catheter angiogram after an appropriate amount span of time, usually three years. If an associated shunt exists, micro-catheter embolization can be performed to achieve the final obliteration. This practical strategy will do help to manage a large AVM containing fistular component.

After going through this article, one would conclude that the role of Gamma knife radiosurgery for AVM in eloquent area should not be exaggerated, especially when the nidal volume is large. Nevertheless, conservative management is not a good treatment option for deep-seated AVM with history of hemorrhage. The policy of 'at least do no harm' should be bear in mind by all means. AVM patients with compact nidus, bleeding history, evidence of impending bleeding or neurological deterioration may be subjected to benefit from Gamma knife radiosurgery. Peri-radiosurgical embolization is potentially helpful for those AVM containing identifiable high-flow fistula components and/or associated intranidal aneurysm or pseudoaneurysm. I would be certainly cautious to recommend Gamma knife radiosurgery alone as a 'standard of care' for these patients. However, with the improvement of knowledge in radiation biology and radiation technology, radiosurgery may become a treatment of choice for those once considered 'inoperable' AVM.

\section{References}

1. Fiehler J, Stapf C. ARUBA-beating natural history in unruptured brain AVMs by intervention. Neuroradiology 2008;50:465-7.

2. Söderman M, Anderson T, Karlsson B, Wallace MC, Edner G. Management of patients with brain arteriovenous malformations. Eur J Radiol 2003;46:195-205.

3. Javalkar V, Pillai P, Vannemreddy P, Caldito G, Ampil F, Nanda A. Gamma knife radiosurgery for arteriovenous malformations located in eloquent regions of the brain. Neurol India 2009;57:617-9

4. Pan DHC, Guo WY, Chung WY, Shiau CY, Chang YC, Wang LW. 
Gamma knife radiosurgery as a single treatment modality for large cerebral arteriovenous malformations. J Neurosurg 2000;93:113-9.

5. Chung WY, Shiau CY, Wu HM, Liu KD, Guo WY, Wang LW, et al. Staged radiosurgery for extra-large cerebral arteriovenous malformations: Method, implementation, and results. J Neurosurg 2008;109:65-72.
6. Luo CB, Guo WY, Teng MM, Chang FC, Pan DH. Embolization of arteriovenous fistula after radiosurgery for multiple cerebral arteriovenous malformations. Kaohsiung J Med Sci 2005;21:571-7.

Accepted on 06-10-2009

Source of Support: Nil, Conflict of Interest: None declared. 